\section{A hands-on practical tutorial on performing meta-analysis with Stata}

doi:10.1136/eb-2014-101967

\begin{abstract}
Statistical synthesis of research findings via meta-analysis is widely used to assess the relative effectiveness of competing interventions. A series of three papers aimed at familiarising mental health scientists with the key statistical concepts and problems in meta-analysis was recently published in this journal. One paper focused on the selection and interpretation of the appropriate model to synthesise results (fixed effect or random effects model) whereas the other two papers focused on two major threats that compromise the validity of meta-analysis results, namely publication bias and missing outcome data. In this paper we provide guidance on how to undertake meta-analysis using Stata, one of the most commonly used software packages for metaanalysis. We address the three topics covered in the previous issues of the journal, focusing on their implementation in Stata using a working example from mental health research.
\end{abstract}

\section{INTRODUCTION}

Systematic reviews and meta-analyses are often considered a reliable source of evidence to inform decisions about the effectiveness and safety of competing interventions. ${ }^{1}$ The validity of the findings from a meta-analysis depends on several factors, such as the completeness of the systematic review, the plausibility of the assumptions made, the risk of bias in the individual studies and the potential for reporting biases. In this paper we focus on the statistical considerations involved in the meta-analysis process and we analyse an example from mental health in Stata. ${ }^{2}$ The theoretical and conceptual considerations of the methods we implement have been covered in recently published articles $^{3-5}$ and we suggest using these papers as companions when reading this manuscript. More specifically, in this paper we present Stata commands:

- To conduct a fixed or a random effects meta-analysis. Prior to carrying out the statistical analyses, meta-analysts should consider the appropriate model (either fixed or random effects) for the specific clinical setting and outcomes of interest and then interpret the result under the light of the magnitude of the between-studies variability (heterogeneity) ${ }^{3} 6$;

- To account for missing outcome data. Participants with missing outcome data may affect both the precision and the magnitude of the meta-analytic summary effect; the latter can occur when the probability of missingness is related to the effectiveness of the interventions being compared ${ }^{5}$;

- To explore and account for publication bias and small-study effects. ${ }^{4}$ Publication bias occurs when publication of research results depends on their nature and direction. ${ }^{7}$ Failure to account for the unpublished studies may lead to biased summary estimates in favour of one of the two competing treatments (ie, usually the active or the newer intervention). ${ }^{8}$

\section{METHODS AND STATA ROUTINES}

In the following sections

- We provide an example of fixed and random effects meta-analysis using the metan command. ${ }^{9}$

- We use the metamiss command ${ }^{10}$ to explore the impact of different assumptions about the mechanism of missing data on the summary effect.
- We employ different approaches and tools to assess whether publication bias is likely to operate using the commands metafunnel, ${ }^{11}$ confunnel, ${ }^{12}$ metatrim ${ }^{13}$ and metabias. ${ }^{14}$

As a working example, we use a systematic review that comprises 17 trials comparing haloperidol and placebo for the treatment of symptoms in schizophrenia. This dataset has been previously used to evaluate the impact of missing data on clinical outcomes ${ }^{15}$ and is originally based on a Cochrane review. ${ }^{16}$ The outcome of interest is clinical improvement and risk ratios (RRs) larger than 1 favour haloperidol over placebo. From each trial we have the following information (table 1):

- Number of participants who responded in the placebo arm (variable rp) and in the haloperidol arm ( $r h$ );

- Number of participants who did not respond in either arm ( fp, fh);

- Number of participants who dropped out and whose outcomes are missing ( $\mathrm{mp}, \mathrm{mh})$.

\section{Performing fixed and random effects meta-analysis and measuring heterogeneity}

Meta-analysis in Stata can be performed using the metan command.

For dichotomous data, the metan command needs four input variables:

\section{- metan $r h$ fh $r p \mathrm{fp}$}

Typing this, the software gives you the summary RR of haloperidol versus placebo using the fixed effect model according to Mantel-Haenszel weights. ${ }^{17}$ The inverse-variance weights can be specified via the option fixedi or randomi for a fixed or random effects analysis, respectively. Changing the estimated effect size is possible by specifying the options or for $\mathrm{OR}$ and rd for risk difference. The option by ( ) allows definition of a grouping variable for the included studies and runs a subgroup analysis.

For continuous data, six input variables are necessary: the total number of participants in each arm, the mean values and the SD for each arm. The option nostandard switches the

Table 1 Number of successes ( $r$ ), failures ( $f$ ) and missing participants (m) for the 17 trials comparing haloperidol and placebo for clinical improvement in schizophrenia

\begin{tabular}{|c|c|c|c|c|c|c|c|}
\hline \multirow[b]{2}{*}{ First author } & \multirow[b]{2}{*}{ Year } & \multicolumn{3}{|c|}{ Haloperidol arm } & \multicolumn{3}{|c|}{ Placebo arm } \\
\hline & & rh & fh & $\mathbf{m h}$ & rp & $\mathrm{fp}$ & $\mathrm{mp}$ \\
\hline Arvanitis & 1997 & 25 & 25 & 2 & 18 & 33 & 0 \\
\hline Beasley & 1996 & 29 & 18 & 22 & 20 & 14 & 34 \\
\hline Bechelli & 1983 & 12 & 17 & 1 & 2 & 28 & 1 \\
\hline Borison & 1992 & 3 & 9 & 0 & 0 & 12 & 0 \\
\hline Chouinard & 1993 & 10 & 11 & 0 & 3 & 19 & 0 \\
\hline Durost & 1964 & 11 & 8 & 0 & 1 & 14 & 0 \\
\hline Garry & 1962 & 7 & 18 & 1 & 4 & 21 & 1 \\
\hline Howard & 1974 & 8 & 9 & 0 & 3 & 10 & 0 \\
\hline Marder & 1994 & 19 & 45 & 2 & 14 & 50 & 2 \\
\hline Nishikawa 82 & 1982 & 1 & 9 & 0 & 0 & 10 & 0 \\
\hline Nishikawa 84 & 1984 & 11 & 23 & 3 & 0 & 13 & 0 \\
\hline Reschke & 1974 & 20 & 9 & 0 & 2 & 9 & 0 \\
\hline Selman & 1976 & 17 & 1 & 11 & 7 & 4 & 18 \\
\hline Serafetinides & 1972 & 4 & 10 & 0 & 0 & 13 & 1 \\
\hline Simpson & 1967 & 2 & 14 & 0 & 0 & 7 & 1 \\
\hline Spencer & 1992 & 11 & 1 & 0 & 1 & 11 & 0 \\
\hline Vichaiya & 1971 & 9 & 20 & 1 & 0 & 29 & 1 \\
\hline
\end{tabular}


estimated effect measure from standardised mean difference to mean difference.

The command gives information on the presence and magnitude of statistical heterogeneity via the $\mathrm{Q}$-test, the $\mathrm{I}^{2}$ measure and the estimate of the heterogeneity variance $\tau^{2}$ (using the 'method of moments estimator'), which are provided in the output results. Although the estimated $\mathrm{I}^{2}$ and $\tau^{2}$ are routinely reported as fixed values, they are not free of uncertainty around the mean estimate. The CI for the $\mathrm{I}^{2}$ measure can be derived using the command heterogi, which requires the input of the $\mathrm{Q}$-statistic of the meta-analysis and the corresponding degrees of freedom (df, the number of studies minus one):

- heterogi $Q$ df $(Q)$

To date the metan command does not provide a CI for the magnitude of heterogeneity $\left(\tau^{2}\right)$. However, it allows assessing the impact of heterogeneity on the summary effect via the predictive interval, which is the interval within which the effect of a future study is expected to lie. ${ }^{18}$ The predictive interval expresses the additional uncertainty induced in the estimates of future studies due to the heterogeneity and can be estimated by adding the rfdist option in metan (under the random effects model).

Many additional options are available (eg, options that handle the appearance of the forest plot), which can be found at the help file of the command (by typing 'help metan').

\section{Exploring the impact of missing outcome data}

Stata has a readily available command called metamiss that enables incorporation of different assumptions for the mechanism of missing outcome data in a meta-analysis. To date the metamiss command can be applied only for dichotomous data but is currently being extended to account for continuous outcomes. ${ }^{19}$ The syntax is similar to the metan command but requires also the number of participants that dropped out from each arm (ie, six input variables are necessary) as well as the desired method of imputing information for the missing data:

- metamiss rh fh mh rp fp mp, imputation_method

In general, we can assume the following scenarios:

- An available case analysis (ACA), which ignores the missing data (option aca) and justifies the missing at random assumption;

- The best-case scenario, which imputes all missing participants in the experimental group as successes and in the control group as failures (option icab);

- The worst-case scenario, which is the opposite of the best-case scenario (option icaw)

The two previous approaches are naïve imputation methods since they do not account properly for the uncertainty in the imputed missing data. Methods that take into account the uncertainty in the imputed data include:

- The Gamble-Hollis analysis, ${ }^{20}$ which inflates the uncertainty of the studies using the results from the best-case and worst-case analyses (option gamblehollis);

- The informative missingness OR (IMOR) model, ${ }^{15} 21$ which relates within each study arm the results from observed and missing participants (options imor () or logimor ()) allowing for uncertainty in the assumed association (sdlogimor ()).

Note that the metamiss command always assumes that the outcome is beneficial; hence for a harmful outcome (eg, adverse events) the options icab and icaw will give the worst-case and best-case scenario, respectively. If it is not possible to assume that missing data are missing at random, the IMOR model is the most appropriate method because it takes the uncertainty of imputed data into account. ${ }^{5}$ This model uses a parameter that relates the odds of the outcome in the missing data to the odds of the outcome in the observed data. If this parameter cannot be informed by expert opinion, it is prudent to conduct a sensitivity analysis assuming various values (eg, if we set the odds of the outcome in the missing data to be twice as much as the odds in the observed data for treatment as well as control groups, we type metamiss $\mathrm{rh}$ fh $\mathrm{mh} \mathrm{rp} \mathrm{fp} \mathrm{mp}$, imor (2)).

\section{Assessing the presence of small study effects and the risk of publication bias}

The available approaches for assessing the risk of publication bias in a meta-analysis can be broadly classified into two categories: (1) methods based on associating effect sizes to their precision and (2) selection models. We focus on the first group of methods, which have been implemented in Stata via the commands metafunnel, confunnel, metatrim and metabias. However, researchers should always remember that this approach gives information about the presence of small study effects, which might or might not be associated with genuine publication bias. ${ }^{4}$

The command metafunnel draws the standard funnel plot $^{22}$ and requires two input variables. Let logRR and selogRR be the two variables containing the observed effect sizes in studies and their SEs. The syntax of the metafunnel command would be:

\section{- metafunnel logRR selogRR}

The option by () can be added to display the studies in subgroups (using different shapes and colors) according to a grouping variable. A limitation of the standard funnel plot is that it does not explain whether the apparent asymmetry is due to publication bias or other reasons, such as genuine heterogeneity between small and large studies or differences in the baseline risk of the participants. ${ }^{4}$ Contour-enhanced funnel plots can be used instead where shaded areas have been added in the graph to indicate whether missing studies lie in the areas of statistical significance (eg, $p$ value $<0.05$ ). ${ }^{23}$ If non-significant studies have been published, it is unlikely that the asymmetry is due to publication bias. The command confunnel can be employed to produce this modified funnel plot using the same syntax with the metafunnel command:

\section{- confunnel logRR selogRR}

The measure of study precision plotted in the vertical axis (eg, the variance instead of the SE) can be modified via the option metric (), while the option extraplot () allows the incorporation of additional graphs (such as regression lines, alternative scatterplots, etc) using standard Stata commands.

Alternatives to funnel plot have also been implemented in Stata. ${ }^{22}$ More specifically, regression models that consider the magnitude of effect in a trial to be related to its precision are very popular. The command metabias can fit four different regression models; these are the Egger's test ${ }^{22}$ (option egger), the Harbord's test ${ }^{24}$ (option harbord), the Peter's test ${ }^{25}$ (option peter) and the rank correlation test by Begg and Mazumdar' ${ }^{26}$ (option begg). For a generic approach where the study effect sizes (effect) are regressed on their standard errors (se)

- metabias effect se, model

or for dichotomous data

- metabias rh fh $\mathrm{rp} \mathrm{fp}$, model

where model defines one of the four models described above. Adding the option graph also gives a graphical depiction of the results. Note that the regression line estimated by the 
Egger's test can also be added to the funnel plot by adding the option egger in the metafunnel command.

The trim-and-fill method aims to estimate the summary effect as if the funnel plot were symmetrical assuming that publication bias is the only explanation of asymmetry. The method can be applied using the metatrim command with the following syntax:

- metatrim effect se

Specifying the option funnel in metatrim gives the estimated filled funnel plot that includes both published and unpublished studies.

\section{RESULTS}

A Stata script that produces all results described below can be found online at http://missoptima.project.uoi.gr/index.php/ our-research-projects.

\section{Fixed and random effects meta-analysis}

We fitted fixed effect as well as random effects models for illustration purposes. Using the metan command, we carried out ACAs for both models and produced the forest plot of figure 1. It is generally misleading to focus on the diamond when interpreting the results of a random effects meta-analysis; for example, in the presence of excessive heterogeneity the diamond is often meaningless.

According to figure 1, both models suggested that haloperidol was statistically significantly more effective than placebo in the treatment of schizophrenia and, as expected, the random effects analysis produced wider CI. Despite this finding, the estimated predictive interval crossed the line of no effect implying that in a future study placebo might appear to be more effective than the active drug. The study-specific estimates seemed substantially heterogeneous (eg, the CIs of the following studies, Bechelli 1983 and Beasley 1996 did not overlap); hence the fixed effect assumption might not be plausible for this dataset. This is supported by the Q-test, which suggested the presence of heterogeneity $(p=0.038)$. The mean of the $I^{2}$ measure, which measures the amount of heterogeneity across studies, suggested the presence of low heterogeneity (41\%). Using the heterogi command we estimated the CI for the $\mathrm{I}^{2}$, which ranged from $0 \%$ to $67 \%$ implying that heterogeneity was potentially null to large, but not excessive.

The two models did not differ only in the level of uncertainty but also with respect to the magnitude of the summary effect. This is very common when there is a small-study effect (ie, there is an association between effect size and study size) because the random effects model assigns relatively larger weights to smaller studies. ${ }^{4}$ Indeed, in our example smaller studies (ie, studies corresponding to smaller squares in figure 1) gave more favourable results for haloperidol, whereas larger studies were closer to the null effect.

\section{Impact of missing outcome data}

We first fitted a subgroup analysis (using metan with the by () option) to investigate whether studies with and without missing data (in both arms) produced different results. This analysis was based only on the observed data and thus, in studies with missing data, the sample size was less than the number of randomised participants. A common misconception about subgroup analyses is that results differ between subgroups when the summary effect for one subgroup is statistically significant and not for the other. However, inference on subgroup differences should be based on an interaction test (ie, the test for subgroup differences implemented also in RevMan -http://tech.cochrane.org/revman) that compares statistically the two subgroup means accounting for their uncertainty. Differences between subgroups can be also identified visually by looking at the overlap of the CIs in their summary estimates. ${ }^{17}$

In figure 2, trials without missing data gave more favourable results for haloperidol than trials with missing data. This disagreement was also statistically significant since $p$ value for the overall test for heterogeneity between subgroups (provided in the output of metan under the fixed effect model) was equal to 0.001 . Hence, it is likely that missing data in trials substantially affected the results; a possible explanation is that there
Figure 1 Forest plot showing the results of fixed and random effects meta-analysis for the 17 trials comparing haloperidol and placebo for clinical improvement in schizophrenia (outcome: response rate) (RR, risk ratio).

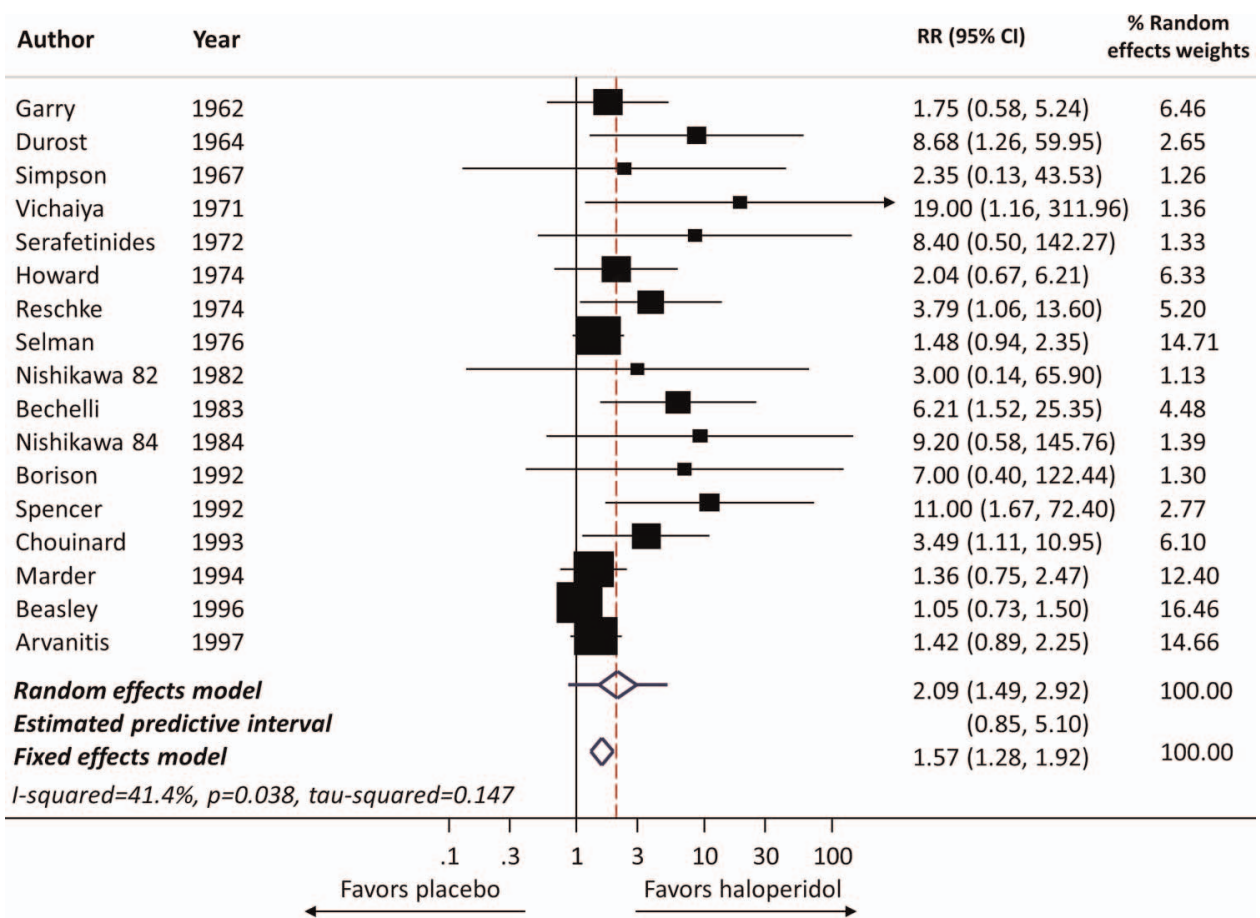


Figure 2 Subgroup analysis of the 17 trials comparing haloperidol and placebo in schizophrenia (outcome: response rate). Studies have been classified according to the presence or absence of missing outcome data in both arms (RR, risk ratio).

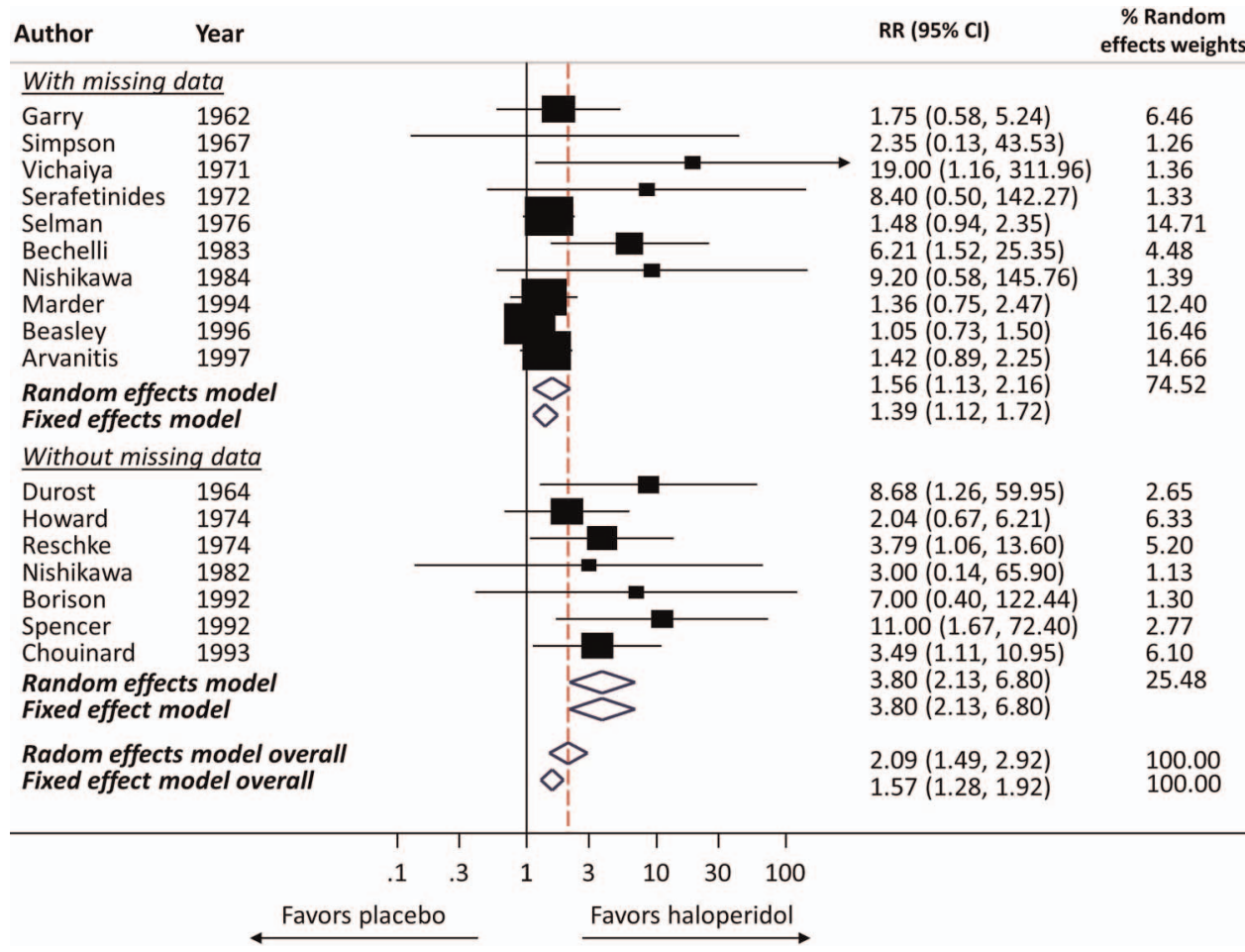

was a high dropout rate in the placebo arm due to lack of efficacy, which is quite common in trials in psychiatry.

We further explored the impact of missing data by incorporating in the analysis different assumptions about the mechanism of missingness. We presented the results from the random effects model (figure 3) and focused on the differences in the summary effects across the different scenarios. Under all six analyses haloperidol appeared to work better than placebo for schizophrenia. Small differences existed in the point estimates between the IMOR models and the Gable-Hollis analysis compared to the ACA. Unlike the rest of the methods these two approaches do not impute data and do not artificially inflate the sample size. The IMOR model increased uncertainty within studies which, in turn, resulted in a slight reduction of heterogeneity. The changes in the summary estimate were negligible. Under the ACA analysis studies with large missing rates favoured placebo (figure 1). The IMOR models down-weighted these studies and the mean summary estimate moved slightly towards the direction of the active intervention.

\section{Publication bias and small-study effects}

Different summary estimates between fixed and random effects models (figure 1) raised concerns that small-study effects possibly operated in our example, questioning the correct interpretation of the overall effect. To explore this apparent association between effect size and study size, we employed a series of graphical approaches and statistical tests (it is important to
Figure 3 Summary risk ratios (RRs) as estimated when the missing data are ignored (available case), by naïve imputation methods (best-case and worst-case) and by methods that properly account for the uncertainty in missing data (Gamble-Hollis and informative missingness OR (IMOR) models). Both IMOR models assume that the odds of the outcome in the missing data are equal to the odds in the observed data (mean IMOR=1) and reflect the uncertainty of this assumption by allowing for a non-zero SD of the missing parameter.
Assumption for missingness

RR (95\% Cl)

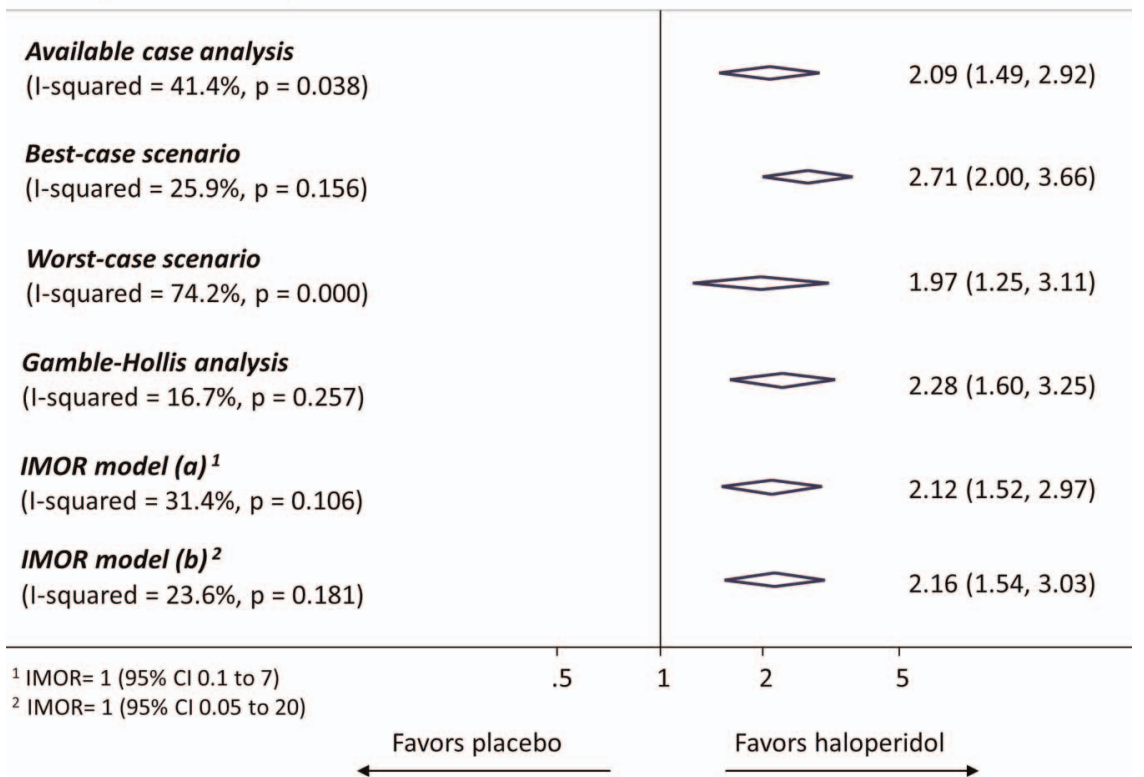




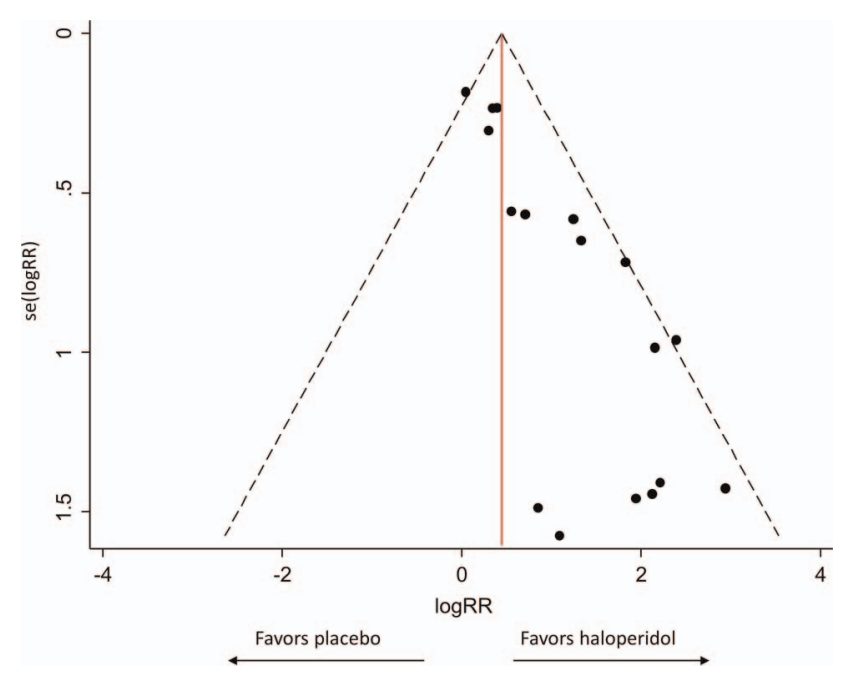

Figure 4 Funnel plot of the 17 trials comparing haloperidol and placebo in schizophrenia (outcome: response rate). The vertical line corresponds to the summary $\log (\mathrm{RR})$ as estimated from the fixed effect model (RR, risk ratio).

note that all these methods have low power and at least 10 studies are needed to draw conclusions). ${ }^{17}$

The funnel plot in figure 4 was rather asymmetric and showed that smaller studies tended to give results emphasising the effectiveness of haloperidol. The contour-enhanced funnel plot (figure 5) helped us distinguish between publication bias and other causes of the asymmetry. It showed that small studies were found not only in the areas of statistical significance (shaded area) but also in areas of non-statistical significance (white area); hence asymmetry might have been caused by several factors and not solely by publication bias. To assess the magnitude and statistical significance of the relationship between observed effect sizes and the size of studies we ran the Egger's meta-regression model (table 2). The test suggested that smaller studies tended to give different results if compared with larger trials, as the CI of the intercept did not include the zero value.

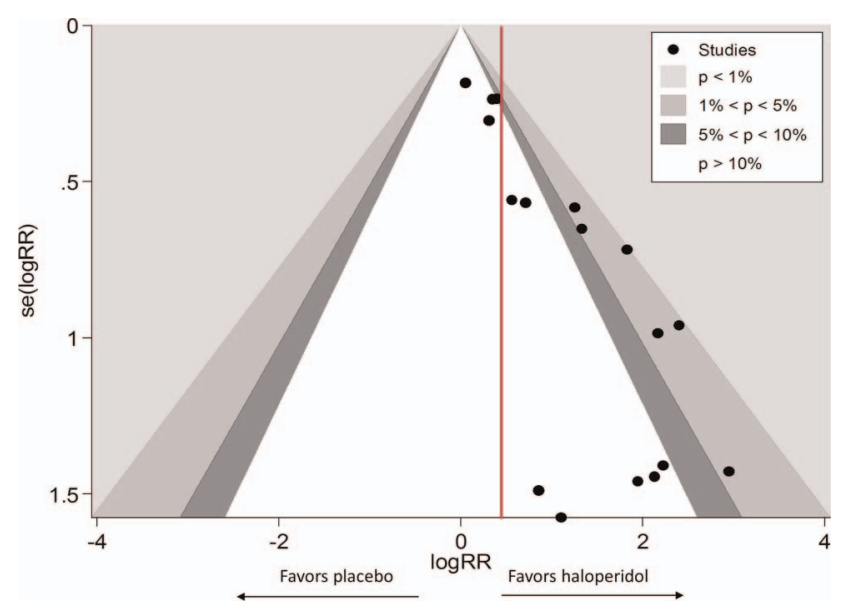

Figure 5 Contour-enhanced funnel plot of the 17 trials comparing haloperidol and placebo for clinical improvement in schizophrenia. The vertical line corresponds to the estimated summary $\log (\mathrm{RR})$. The shaded areas correspond to levels of statistical significance defined by the $\mathrm{p}$ value of a $\mathrm{z}$-test for the $\log \mathrm{RR}$ ( $\mathrm{RR}$, risk ratio).
Table 2 Results from the Egger's meta-regression test assessing the presence of small-study effects in the 17 trials comparing haloperidol and placebo for clinical improvement in schizophrenia

\begin{tabular}{lccccrl}
\hline Parameter & Estimate & SE & t Value & p Value & $\mathbf{9 5 \%} \mathbf{~ C l}$ & \\
\hline Slope (coefficient) & -0.15 & 0.11 & -1.34 & 0.20 & -0.38 & 0.09 \\
Bias (intercept) & 1.79 & 0.26 & 6.95 & 0.00 & 1.24 & 2.34 \\
Test of $H_{0}:$ no small-study effects, & $p$ value $=0.000$ & & & \\
\hline
\end{tabular}

We finally applied the trim-and-fill method, although the assumption that funnel plot asymmetry was solely caused by publication bias probably did not hold for this dataset. The addition of the nine estimated unpublished studies slightly moved the summary estimate of the fixed effect model closer to 0 (figure 6), while the random effects model (table 3) resulted in a non-significant summary estimate $(p=0.083)$.

\section{DISCUSSION}

Along with the rapid methodological development of meta-analysis, a variety of relevant software options have been made available enabling the application of different models and the exploration of characteristics that may affect the results. Using a working example, in this paper we offered a brief tutorial to researchers and interested clinicians about the use of Stata in meta-analysis, highlighting common pitfalls in the interpretation of results (more information about Stata can be found elsewhere). ${ }^{27}$ Our findings suggested that the presence of important small-study effects as well as the missing outcome data in some trials made the estimated summary effect not representative for the entire set of studies. Including in the meta-analysis only studies with data for all randomised participants was not the recommended approach, since the bulk of evidence came from trials with missing outcome data.

Clinical insight for the outcomes and treatments of interest is necessary to make reasonable assumptions for the mechanism of missing data and inform the choice of the appropriate statistical model. The results from the three models that accounted for the uncertainty in the imputed missing data (Gamble-Hollis and the two IMOR models) were similar and probably are the most accurate estimates of the summary RR.

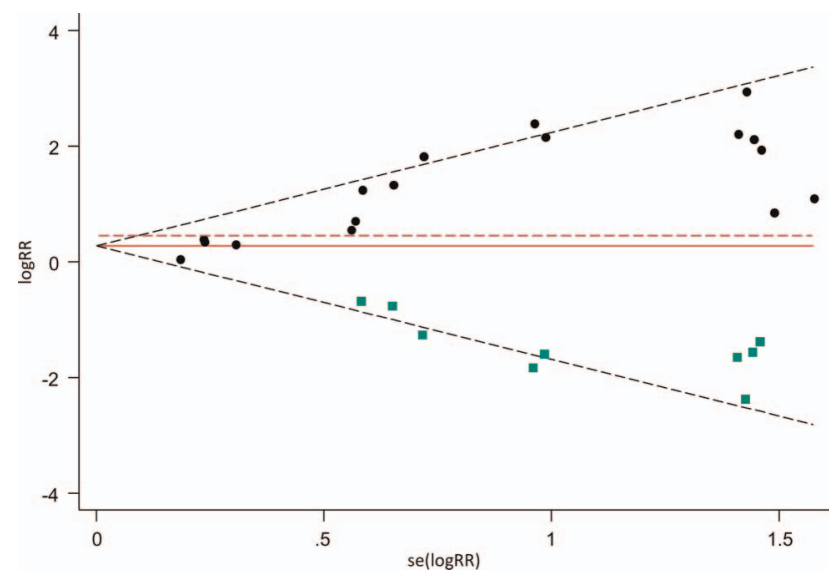

Figure 6 Funnel plot including the 17 published haloperidol trials in schizophrenia (circles) and the unpublished studies (squares) as estimated from the trim-and-fill method. The solid line corresponds to the adjusted for the impact of publication bias summary effect $(\log R R=0.27)$ and the dashed line to the summary effect that does not account for publication bias (logRR $=0.45$ ) ( $R R$, risk ratio). 
Table 3 Results from the trim-and-fill method for publication bias in the 17 haloperidol trials for schizophrenia

\begin{tabular}{llrllll}
\hline \multicolumn{1}{l}{ Filled meta-analysis } \\
\hline & $\begin{array}{l}\text { Summary } \\
\text { logRR }\end{array}$ & $\mathbf{9 5 \%} \mathbf{~ C l}$ & & z Value & p Value & $\begin{array}{l}\text { Number } \\
\text { of studies }\end{array}$ \\
Model & 0.274 & 0.084 & 0.465 & 2.825 & 0.005 & 26 \\
\hline $\begin{array}{l}\text { Fixed effect } \\
\begin{array}{l}\text { Random } \\
\text { effects }\end{array}\end{array}$ & 0.331 & -0.044 & 0.705 & 1.731 & 0.083 & 26 \\
\hline
\end{tabular}

$\mathrm{RR}$, risk ratio.

However, the fact small and large trials gave different results needs further exploration. For example, if the size of the studies was associated with differences in population characteristics with respect to some effect modifiers, then there might not be a common RR applicable to all populations.

\section{Anna Chaimani, ${ }^{1}$ Dimitris Mavridis, ${ }^{1,2}$ Georgia Salanti ${ }^{1}$}

${ }^{1}$ Department of Hygiene and Epidemiology, University of loannina School of Medicine, Ioannina, Greece

${ }^{2}$ Department of Primary Education, University of Ioannina, Ioannina, Greece

Correspondence to: Dr Anna Chaimani, achaiman@cc.uoi.gr

Competing interests $\mathrm{AC}$ received funding from Greek national funds through the Operational Program "Education and Lifelong Learning" of the National Strategic Reference Framework (NSRF)-Research Funding Program: ARISTEIA. Investing in knowledge society through the European Social Fund. DM and GS received funding from the European Research Council (IMMA 260559).

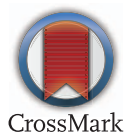

\section{REFERENCES}

1. Cipriani A, Furukawa TA. Advancing evidence-based practice to improve patient care. Evid Based Ment Health 2014;17:1-2.

2. StataCorp. Stata Statistical Software. College Station, TX: StataCorp LP, 2013.

3. Nikolakopoulou A, Mavridis D, Salanti G. Demystifying fixed and random effects meta-analysis. Evid Based Ment Health 2014;17:53-7.

4. Mavridis D, Salanti G. Exploring and accounting for publication bias in mental health: a brief overview of methods. Evid Based Ment Health 2014;17:11-15.

5. Mavridis D, Chaimani A, Efthimiou 0, et al. Addressing missing outcome data in meta-analysis. Evid Based Ment Health 2014;17:85-9.
6. Borenstein $\mathbf{M}$, Hedges LV, Higgins JPT, et al. A basic introduction to fixed-effect and random-effects models for meta-analysis. Res Synth Methods 2010;1:97-111.

7. Dickersin K. The existence of publication bias and risk factors for its occurrence. JAMA 1990;263:1385-9.

8. Turner EH, Matthews AM, Linardatos E, et al. Selective publication of antidepressant trials and its influence on apparent efficacy. N Engl J Med 2008:358:252-60.

9. Harris R, Bradburn M, Deeks J, et al. Metan: fixed-and random-effects meta-analysis. Stata J 2008;8:3.

10. White IR, Higgins JP. Meta-analysis with missing data. Stata J 2009;9:57

11. Sterne JA, Harbord RM. Funnel plots in meta-analysis. Stata J 2004;4: 127-41.

12. Palmer TM, Peters JL, Sutton AJ, et al. Contour-enhanced funnel plots for meta-analysis. Stata J 2008:8:242.

13. Steichen TJ. Nonparametric trim and fill analysis of publication bias in meta-analysis: erratum. Stata Tech Bull 2001;10. http://econpapers.repec.org/article/ tsjstbull/y_3a2001_3av_3a10_3ai_3a58_3asbe39.1.htm (accessed 3 Sep 2014).

14. Harbord $\mathbf{R} \mathbf{M}$, Harris RJ, Sterne JAC. Updated tests for small-study effects in meta-analyses. Stata J 2009;9:197-210.

15. Higgins JP, White IR, Wood AM. Imputation methods for missing outcome data in meta-analysis of. Clin Trials 2008:5:225-39.

16. Joy CB, Adams CE, Lawrie SM. Haloperidol versus placebo for schizophrenia Cochrane Database Syst Rev 2006;(4):CD003082.

17. Deeks JJ, Higgins JP, Altman DG. Analysing data and undertaking meta-analyses. In: Higgins JPT, Green S. eds. Cochrane handbook for systematic reviews of interventions. John Wiley \& Sons, Ltd, 2008:243-96.

18. Riley RD, Higgins JPT, Deeks JJ. Interpretation of random effects meta-analyses. BMJ 2011;342:d549.

19. Mavridis D, White IR, Higgins JPT, et al. Addressing continuous missing outcomes in pairwise and network meta-analysis. 35th ISCB Annual Conference; Vienna, August 2014. http://www.mtm.uoi.gr/index.php/research-and-publications/ 11-rap-articles/22-talks

20. Gamble C, Hollis S. Uncertainty method improved on best-worst case analysis in a binary meta-analysis. J Clin Epidemiol 2005;58:579-88.

21. White IR, Higgins JPT, Wood AM. Allowing for uncertainty due to missing data in meta-analysis - part 1: two-stage methods. Stat Med 2008;27:711-27.

22. Egger M, Davey Smith G, Schneider M, et al. Bias in meta-analysis detected by a simple, graphical test. BMJ 1997;315:629-34.

23. Peters JL, Sutton AJ, Jones DR, et al. Contour-enhanced meta-analysis funnel plots help distinguish publication bias from other causes of asymmetry. J Clin Epidemiol 2008;61:991-6.

24. Harbord RM, Egger M, Sterne JAC. A modified test for small-study effects in meta-analyses of controlled trials with binary endpoints. Stat Med 2006;25: 3443-57.

25. Peters JL, Sutton AJ, Jones DR, et al. Comparison of two methods to detect publication bias in meta-analysis. JAMA 2006;295:676-80.

26. Begg CB, Mazumdar M. Operating characteristics of a rank correlation test for publication bias. Biometrics 1994;50:1088-101.

27. Sterne J. ed. Meta-analysis in Stata: an updated collection from the Stata Journal. Stata Press, 2009. http://www.libreriasaulamedica.com/ficha.asp?id=69107 (accessed 3 Sep 2014). 